\title{
Demonstration Abstract: Research Platform for Visible Light Communication and Sensing Systems
}

\author{
Qing Wang ${ }^{1,2}$ Danilo De Donno ${ }^{1} \quad$ Domenico Giustiniano $^{1}$ \\ ${ }^{1}$ IMDEA Networks Institute, Madrid, Spain $\quad{ }^{2}$ University Carlos III of Madrid, Spain \\ Email: \{qing.wang, danilo.dedonno,domenico.giustiniano\}@imdea.org
}

\begin{abstract}
OpenVLC (www.openvlc.org) is an open-source project for research in Visible Light Communication (VLC) systems. It has the potential to help create a new type of infrastructure, an Internet of Lights (IoL), where LED-based devices (e.g., car lights, city lights, billboards, toy, etc) and photodetectors become inter-connected. OpenVLC is built upon an embedded platform and adopts off-the-shelf optical devices and essential electronic components. It has been proved to be a starter kit for VLC research, thanks to its deployments by tens of top universities/research centers in the world (www.openvlc.org/list-of-users.html). This demo introduces its latest version: OpenVLC1.1. The new features include: (1) higher resilience to ambient light noise, e.g., indoor interfering lighting; (2) interface for sensor application. A new board (OpenVLC1.1 cape) is designed and the printed circuit is developed. The cape is plugged directly into the main embedded board and external sensors can be easily connected to the cape. This demo demonstrates OpenVLC1.1's networking performance through standard networking diagnostic tools and shows an application wherein temperature and humidity sensed data are transmitted through VLC links.
\end{abstract}

Index Terms-Visible light communication; OpenVLC; Opensource; Low-cost; Research platform; Sensors

\section{INTRODUCTION}

Visible Light Communication (VLC) is an emerging technology that has attracted the attention of researchers for accurate indoor localization [1], clock calibration [2], Internet of Things (IoT) [3], [4], [5], [6], and next-generation high-speed cellular networks [7]. As there are an increasing number of devices embedding LEDs, e.g., home appliances, smartphone, cars, city lights, billboard, etc., in the future we may have a new type of infrastructure waiting to be networked, an Internet of Lights (IoL). A step in this direction is our OpenVLC platform [8], an open-source project with low-cost hardware designed for rapid prototyping of embedded networked VLC systems.

This demo introduces the latest OpenVLC1.1 platform, that increases the resilience to ambient light noise, e.g., indoor interfering lighting (thus it can now work under normal illumination interfering lights), and introduces an interface for sensor application. A new printed circuit board -OpenVLC1.1 cape (Fig. 1) - has been designed and developed. The cape implements the VLC transceiver and it is plugged directly into the main embedded system board board. This feature allows researchers to focus on the software design. Researchers can also easily attach additional sensors to the OpenVLC1.1 board to use VLC as a new communication medium for IoT devices

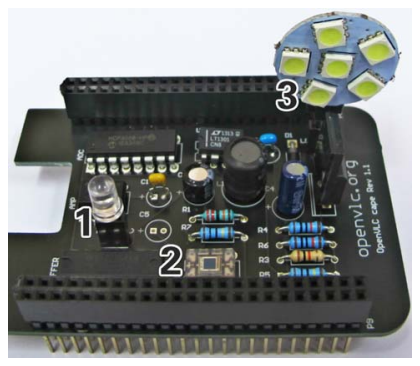

(a) The OpenVLC1.1 cape

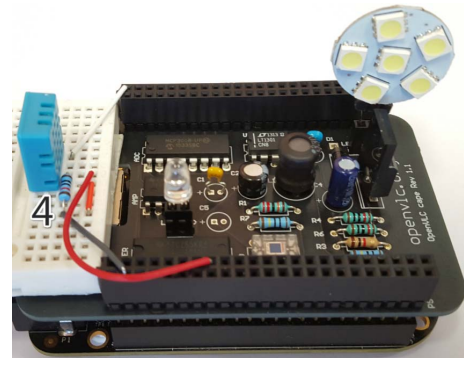

(b) Sensors connected to the cape. The cape is plugged into the embedded system board.
Fig. 1. An OpenVLC1.1 node. The (optical) components are: (1) low-power LED; (2) photodiode; (3) high-power LED. A temperature and humidity sensor (4) is attached to the cape.

and build various applications. The cape is controlled using the OpenVLC1.1 driver, that implements key primitives at the MAC and PHY layers such as signal sampling, symbol detection, coding/decoding, channel contention, carrier sensing and Internet protocol interoperability [4]. OpenVLC1.1 provides the basic tools to implement various protocols and applications, and prototype them in real-world VLC network setups.

\section{SYSTEM DESIGN}

OpenVLC1.1 is a general-purpose, software-defined platform. The block diagram of the front-end transceiver is shown in Fig. 2(right). OpenVLC1.1 implements a front-end transceiver that adopts a high-power LED, a low-power LED, and a photodiode (PD) to transmit and receive lights. It can select via software the transmitter (TX) on-the-fly between the high-power and low-power LEDs. Similarly, it can select the receiver $(\mathrm{RX})$ between the $\mathrm{PD}$ and low-power LED.

Several VLC links are available based on the three optical antennas of OpenVLC1.1:

- low-/high-power LED-to-PD communication;

- low-power LED-to-LED communication;

- high-power LED to low-power LED communication

Each configuration of TX/RX comes with its own unique features in terms of channel propagation, RX sensitivity, Fieldof-View (FoV), etc. Flexible protocols can be designed that can dynamically choose the most desired configuration based on the environmental conditions and the application. 


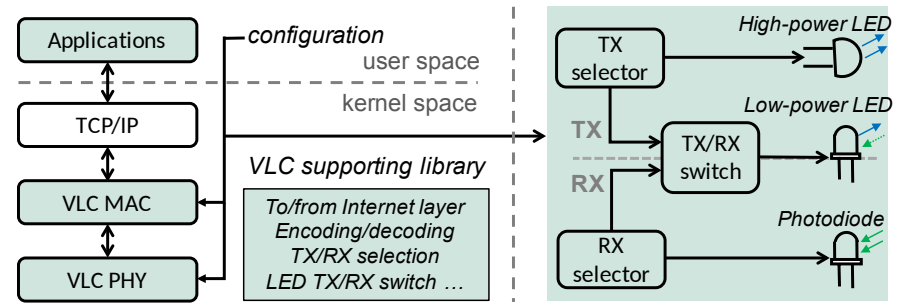

Fig. 2. Block diagrams of the OpenVLC1.1: left) implementation of the software stack in the Linux operating system (OpenVLC1.1 driver); right) flexible front-end transceiver (OpenVLC1.1 cape).

The software stack is shown in Fig. 2(left). The software solutions of OpenVLC1.1 are implemented as a Linux driver (new network interface) that can be connected to TCP/IP layers. As such, traditional networking diagnostic tools such as ping and iperf can be easily adopted to evaluate OpenVLC1.1's performance. Furthermore, developing applications that use the visible light as transmission medium becomes straightforward because applications only have to simply specify OpenVLC1.1 network interface.

\section{DEMONSTRATION}

The embedded system board (BeagleBone, $\mathrm{BBB}^{1}$ ) runs the Debian Linux operating system with kernel version 3.8.13 and the Xenomai patch. The cape adopts off-the-shelf electronic devices. At the PHY layer, we implement On-Off-Keying modulation with Manchester code and Reed-Solomon error correcting code. To ensure a fair channel access among nodes and reduce transmission collisions, we implement three MAC protocols:

- listen-before-talk Carrier Sensing Multiple Access with Collision Avoidance (CSMA/CA);

- listen-while-talk CSMA with Collision Detection (CSMA/CD) [3];

- listen-before\&while-talk CSMA with Collision Detection and Hidden Avoidance (CSMA/CD-HA) [9].

We demonstrate the functionalities of OpenVLC1.1 through the following applications:

(I) Standard networking diagnostic tools. We demonstrate the network layer performance of OpenVLC1.1 through wellknown networking diagnostic tools ping and iperf using two nodes, with indoor illuminating lights on. We show the network connectivity by letting one node pings the other; and demonstrate the achievable UDP throughput with iperf.

(II) Customized sensory application. A high-level representation of this application is illustrated in Fig. 3. We transmit sensed temperature\&humidity data using VLC links. The data received is shown to the audience with a web browser application. We use two OpenVLC1.1 nodes, a temperature\&humidity sensor, and a laptop:

- Node A: it periodically probes the temperature and humidity sensor (model DHT11). These data are then copied

${ }^{1}$ http://beagleboard.org/Products/BeagleBone+Black

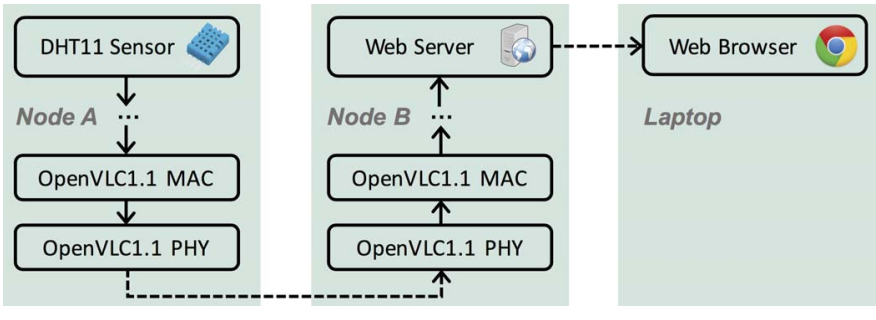

Fig. 3. Illustration of the customized application that uses VLC as a new communication medium to communicate the data gathered by a sensor.

to the VLC MAC layer and transmitted to Node B through VLC link (it can choose any available VLC link from those presented in Section II).

- Node B: it decodes the received data from Node A and then submits the decoded data of temperature and humidity to the web server that runs in Node B;

- Laptop: it visually shows the temperature\&humidity output at the receiving node $\mathrm{B}$ to the audience with a web browser application.

\section{CONCLUSION}

In this demo, we introduced the OpenVLC1.1 research platform for the Internet of Lights. We showcased its capabilities through standard networking diagnostic tools and through a customized sensor application. We invite research groups interested in the OpenVLC1.1 cape to submit a research project to our website www.openvlc.org.

\section{ACKNOWLEDGEMENT}

We thank Omprakash Gnawali (University of Houston) to help us distribute the OpenVLC capes. This work was funded in part by Ministerio de Economía y Competitividad grant TEC2014-55713-R and partially by the Madrid Regional Government through the TIGRE5-CM program (S2013/ICE2919).

\section{REFERENCES}

[1] Y.-S. Kuo, P. Pannuto, K.-J. Hsiao, and P. Dutta, "Luxapose: Indoor positioning with mobile phones and visible light," in ACM MobiCom, 2014, pp. 447-458.

[2] Z. Li, W. Chen, C. Li, M. Li, and etc., "Flight: Clock calibration using fluorescent lighting," in ACM MobiCom, 2012, pp. 329-340.

[3] D. Giustiniano, N. Tippenhauer, and S. Mangold, "Low-complexity visible light networking with LED-to-LED communication," in IFIP Wireless Days, 2012, pp. 1-8.

[4] Q. Wang, D. Giustiniano, and D. Puccinelli, "An open-source research platform for embedded visible light networking," IEEE Wireless Coтmunication, vol. 22, no. 2, pp. 94-100, 2015.

[5] J. Li, A. Liu, and etc., "Retro-VLC: Enabling Battery-free Duplex Visible Light Communication for Mobile and IoT Applications," in ACM HotMobile, 2015, pp. 21-26.

[6] Q. Wang, D. Giustiniano, and O. Gnawali, "Low-cost, flexible and open platform for visible light communication networks," in ACM HotWireless, 2015, pp. 31-35.

[7] D. Tsonev, S. Videv, and H. Haas, "Towards a $100 \mathrm{~Gb} / \mathrm{s}$ visible light wireless access network," Optics Express, 2015.

[8] Q. Wang, D. Giustiniano, and D. Puccinelli, "OpenVLC: Software-defined Visible Light Embedded Networks," in ACM VLCS, 2014, pp. 15-20.

[9] Q. Wang and D. Giustiniano, "Intra-Frame Bidirectional Transmission in Networks of Visible LEDs," Accepted by IEEE/ACM Transactions on Networking, pp. 1-13, 2016. 